LAWRENCE LIVERMORE NATIONAL LABORATORY

Design Considerations of Fast Kicker Systems for High Intensity Proton Accelerators

W. Zhang, J. Sandberg, W. M. Parsons, P. Walstrom, M. M. Murray, E. Cook, E. Hartouni

June 12, 2001

2001 IEEE International Pulsed Power Plasma Science Conference, Las Vegas, NV June 17-22, 2001 
This document was prepared as an account of work sponsored by an agency of the United States Government. Neither the United States Government nor the University of California nor any of their employees, makes any warranty, express or implied, or assumes any legal liability or responsibility for the accuracy, completeness, or usefulness of any information, apparatus, product, or process disclosed, or represents that its use would not infringe privately owned rights. Reference herein to any specific commercial product, process, or service by trade name, trademark, manufacturer, or otherwise, does not necessarily constitute or imply its endorsement, recommendation, or favoring by the United States Government or the University of California. The views and opinions of authors expressed herein do not necessarily state or reflect those of the United States Government or the University of California, and shall not be used for advertising or product endorsement purposes. 


\title{
DESIGN CONSIDERATIONS OF FAST KICKER SYSTEMS FOR HIGH INTENSITY PROTON ACCELERATORS ${ }^{1,2}$
}

\author{
W. Zhang, J. Sandberg \\ Brookhaven National Laboratory, C-A Dept \\ Upton, $N Y$, USA \\ W. M. Parsons, P. Walstrom, M. M. Murray \\ Los Alamos National Laboratory, LANSCE Division \\ Los Alamos, NM, USA \\ E. Cook, E. Hartouni \\ Lawrence Livermore National Laboratory \\ Livermore, CA, USA
}

\begin{abstract}
In this paper, we discuss the specific issues related to the design of the Fast Kicker Systems for high intensity proton accelerators. To address these issues in the preliminary design stage can be critical since the fast kicker systems affect the machine lattice structure and overall design parameters. Main topics include system architecture, design strategy, beam current coupling, grounding, end user cost vs. system cost, reliability, redundancy and flexibility. Operating experience with the Alternating Gradient Synchrotron injection and extraction kicker systems at Brookhaven National Laboratory and their future upgrade is presented. Additionally, new conceptual designs of the extraction kicker for the Spallation Neutron Source at Oak Ridge and the Advanced Hydrotest Facility at Los Alamos are discussed.
\end{abstract}

\section{INTRODUCTION}

Pulsed-power technology has grown rapidly in the area of particle accelerators. Fast manipulation of high energy beams is often accomplished by the application of high-strength, pulsed electromagnetic fields. For instance, in the Brookhaven National Laboratory's (BNL) Collider-Accelerator Complex, we use pulsedpower technology for fast injection and extraction kickers, injection and extraction septa, orbit bumps, gamma-transition energy jumps, and beam instrumentation. Advances in pulsed-power technology are needed for upgrades of existing accelerators, new accelerators under construction, and future accelerators in the design and proposal stage. These advances frequently include combinations of higher peak powers, faster repetition rates, shorter pulse rise/fall times, tighter pulse repeatability, more precise wave shapes, multi-purpose and multi-mode applications, and improved flexibility and reliability.

Of all the pulsed power systems used in accelerator facilities, the fast kicker systems have been the most challenging ones to design, develop, implement and operate. Circuit designers are faced with a very limited choice of devices and must often rate them at levels beyond the manufacturer's specifications. Due to the cost impact, physical size, and the performance requirements, kicker systems have become an increasingly important part of an overall accelerator project. It is therefore necessary to address the kicker design and evaluate possible options in the early stages of lattice design and project planning.

\section{NEW DESIGNS AND PROPOSALS}

\section{A. Alternating Gradient Synchrotron Fast Kicker System Upgrade}

A picture of the Collider-Accelerator Complex,

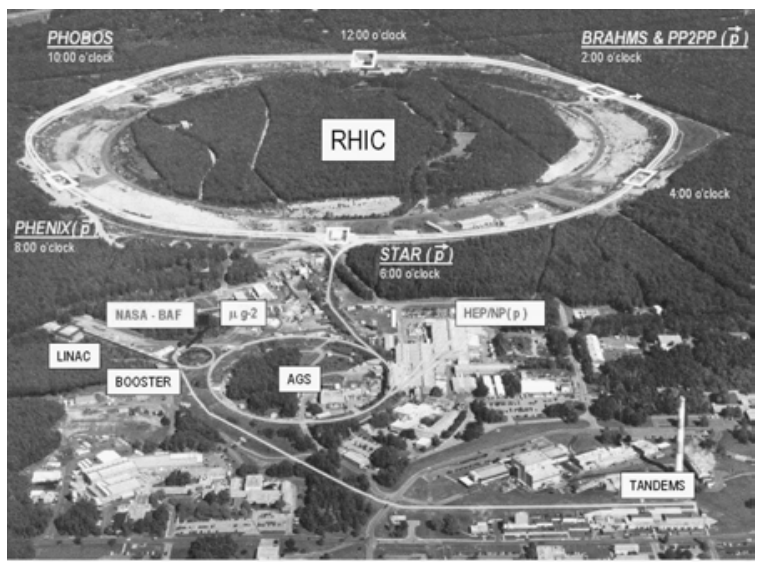

Figure 1. Collider-Accelerator complex at BNL

\footnotetext{
${ }^{1}$ Work performed under the auspices of the U.S. Dept. of Energy.

${ }^{2}$ This work was performed under the auspices of the U.S. Department of Energy by University of California Lawrence Livermore National Laboratory under Contract No. W-7405-Eng-48.
} 
illustrating the location of the Alternating Gradient Synchrotron (AGS) at BNL, is shown in Fig. 1.

We are currently involved in the conceptual design of an upgrade of the AGS A5 injection kicker system. This upgrade would allow an increase in transfer energy from 1.5 to $2.0 \mathrm{GeV}$. To not affect adjacent beam bunches, the system requires a fast rise and an equally fast fall time of $140 \mathrm{nS}$ and must operate for multiple beam transfers between the Booster and the AGS. It is also desirable to have a variable pulse length. Our design study, based on an external pulser using a transmission line to transport pulses to an existing magnet, has shown that a minimum of $67 \mathrm{kV}$ will be needed to achieve the $2 \mathrm{GeV}$ beam injection. To reduce the operation voltage of the kicker, we are considering using the present A5 section with an additional straight section to extend the kicker magnetic length.

There are several other kicker systems at the BNL Collider-Accelerator Complex being studied for new and upgraded designs. One is the AGS G10 fast extraction kicker system upgrade. This system would be used for multiple extractions of single bunches. It would also be capable of full-turn, multi-bunch extractions. Present and future users of AGS G10 include the Relativistic Heavy Ion Collider (RHIC) yellow and blue rings, the G-2 experiment and other high energy physics experiments.

\section{B. The SNS Extraction Kicker Conceptual Design}

The Spallation Neutron Source (SNS), shown in Fig. 2, is being build at Oak Ridge National Laboratory (ORNL). When completed, it will be a new research facility for neutron science designed and constructed by a collaboration of six DOE laboratories.

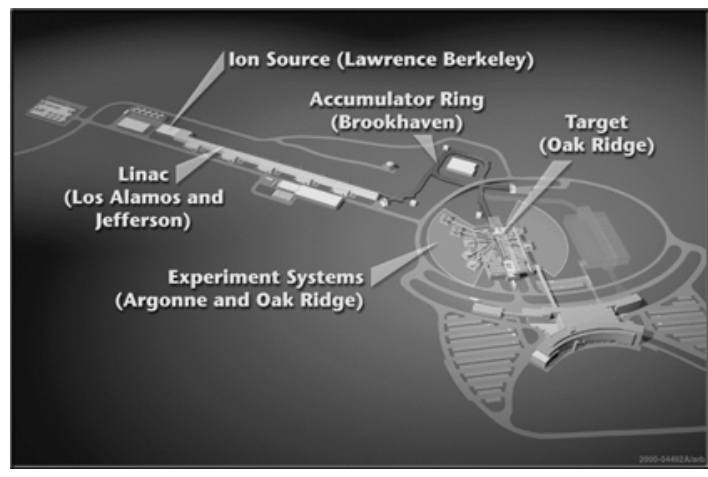

Figure 2. Spallation Neutron Source at ORNL

Its main ring is an OMEGA configuration where one side of the ring is dedicated to beam extraction. The fast kicker system will consist of fourteen magnet sections and fourteen high power modulators. In response to SNS Project Office's recent decision to move all modulators outside of the ring tunnel, and the Accelerator System Review Committee's suggestion of a low beam-impedance design, we proposed a new design that satisfies all system requirements [1].

In the new conceptual design, we use a Blumlein pulser with a parallel resistor and two transmission cables to produce current doubling at the magnet. The maximum voltage will be $50 \mathrm{kV}$ with a peak output current of $4 \mathrm{kA}$ per modulator. A circuit diagram of this concept and the load current waveforms of its $10 \%$ scale model are illustrated in Fig. 3.

The advantages of the new design includes a lower operating voltage of about $25 \mathrm{kV}$, lower beam impedance, largely increased design margin, and substantially fewer transmission cables and tunnel penetrations. With this design, only two cable terminations per module are required inside the ring. To implement the design, it is very critical to have a low inductance assembly.

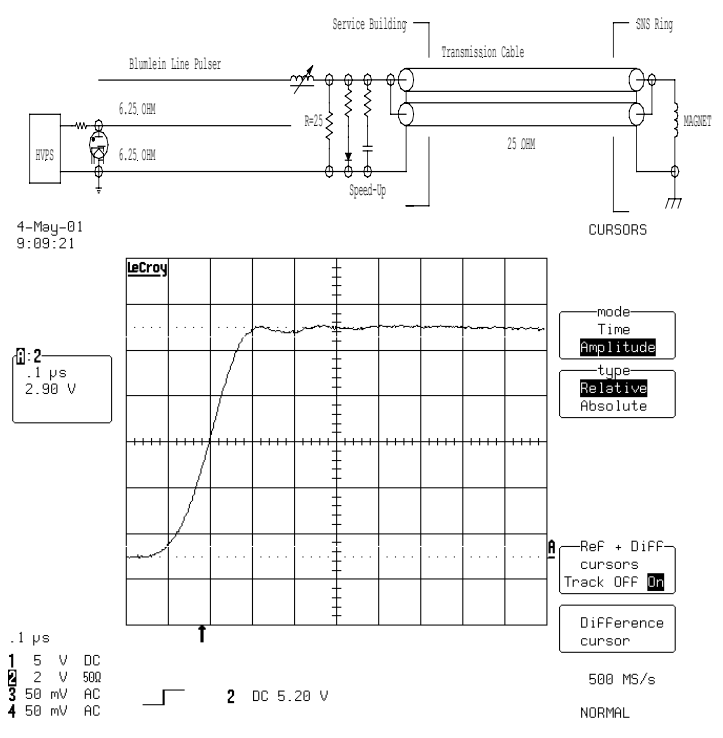

Figure 3. SNS extraction kicker

Top - circuit diagram

Bottom - magnet current waveform of $10 \%$ scale test model

A $10 \%$ scale model circuit has been built as a proof-of-principle. The results demonstrate the design principle works well, even with a double-deck lumped-capacitor PFN for the Blumlein structure, provided the stray inductance of the circuit and its components is kept low. This project is in the first article construction phase.

\section{The Advanced Hydrotest Facility Kicker Systems}

The Advanced Hydrotest Facility (AHF) is a multiaxis, proton-radiography accelerator that is currently in the pre-conceptual design phase at Los Alamos National Lab. The current design has two synchrotron rings: a booster that accumulates a linac beam energy in the range of a few hundred $\mathrm{MeV}$ and accelerates it to $3 \mathrm{GeV}$, and a main synchrotron that supplies protons with maximum energy of $50 \mathrm{GeV}$ to the beam distribution system. Injection into the booster ring will be done by foil-stripping $\mathrm{H}^{-}$ions into protons. The booster synchrotron will therefore require only an 
extraction kicker system. It is believed at present that no abort kicker is needed for the booster.

The main $50-\mathrm{GeV}$ ring will require three separate kicker systems: an injection kicker system, an abort kicker system, and an extraction kicker system. The injection and extraction kickers will operate at a fixed voltage. The modulator voltage for the abort kicker will be ramped with the beam energy, because it must provide the appropriate kick to remove the beam from the ring at any time during the acceleration cycle.

The modulator for the $50-\mathrm{GeV}$ ring-extraction kicker, because of the requirement for multiple pulses at arbitrary times (together with relatively short rise and fall times), is by far the most technically challenging of the modulators. Work on the first stage of the extraction-kicker modulator was started in early 2001 at Lawrence Livermore National Lab. The design is based on an inductive voltage adder topology at $50 \mathrm{kV}$ using $800 \mathrm{~V}$ power MOSFETs as the switching elements. Rise and fall times in the $10-20$ ns range have been demonstrated at $20 \mathrm{kV}$ on a similar unit shown undergoing testing in Fig. 4.

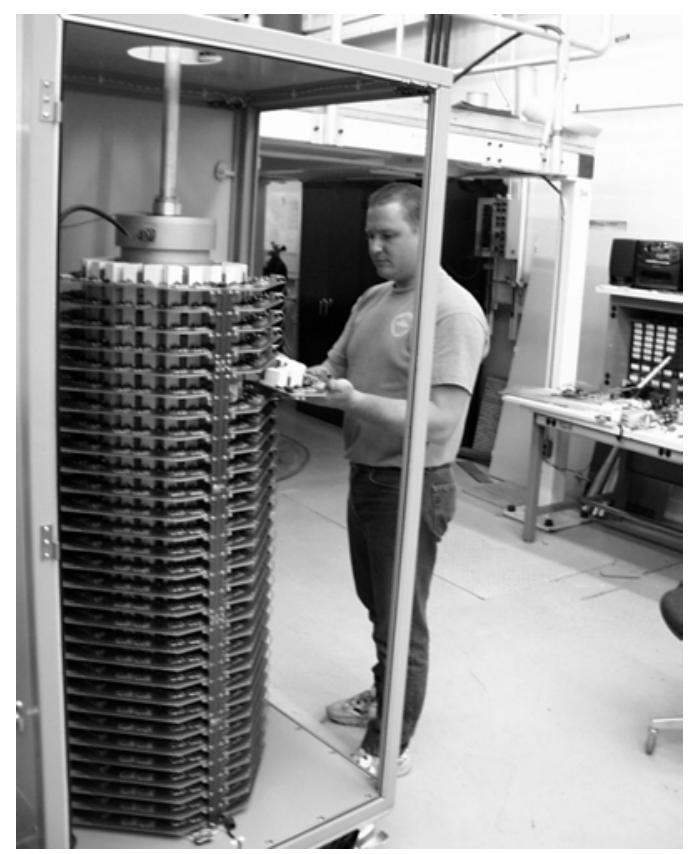

Figure 4. 20-kV kicker modulator under test

\section{DESIGN STRATEGY AND CONSIDERATIONS}

The building blocks of a typical kicker system include an energy source, energy storage and modulation, controlled energy release, energy transport, and deflection plates or magnets.

The deflection mechanism can be pulsed electric field, pulsed magnetic field or a combination of the two. In general, the magnetic field deflection is more efficient than the electric field deflection. Therefore, most high strength kicker systems utilize the travelling-wave magnet or lumped magnet. The magnetic kicker strength is measured by the product of its magnetic field strength and the kicker's effective length. The magnetic field strength is proportional to the current in the kicker magnet. Therefore, most kicker designs use the maximum available longitudinal space to relax the current and voltage requirements on the modulators.

As noted by G. H. Rees [2], extraction at higher energies may lead to more complex hardware development. Therefore, extraction systems often strongly influence the overall design of a machine. For high momentum machines, even the injection energy can be high enough to influence the machine layout. The new designs such as SNS and AHF have allocated long straight sections for fast kicker systems. For instance, the SNS extraction kicker design has a total magnetic length of $6.72 \mathrm{~m}$ and uses 14 modulators, producing a total deflection angle of 74.7 milli-radian to extract the $1.0 \mathrm{Gev}$ proton beam.

In the existing facilities like AGS, we plan to use more straight sections to accommodate kicker upgrade requirements. The existing AGS injection kicker system has a magnetic length of only $0.95 \mathrm{~m}$, and its complimentary extraction kicker system in the AGS booster, commissioned in 1991, has a magnetic length of $2.3 \mathrm{~m}$. The AGS extraction fast kicker has a $2.41-\mathrm{m}$ magnetic length, while the newer RHIC injection kicker system has a magnetic length of $5.4 \mathrm{~m}$. The trend of having longer longitudinal space for fast kicker systems is due to recognizing that kickers are the bottleneck in many accelerators.

In general, key areas important to the design of new fast magnetic kicker systems can be summarized as:

- A $90^{\circ}$ phase shift between kicker and septum;

- Larger $\beta$ values at both kicker and septum locations;

- A thinner septum;

- Longer straight sections for kicker;

- Multiple kicker modules;

- Narrower magnet window width;

- Maximum available pulse rise/fall time;

Other issues, such as beam coupling, grounding and shielding, and components and materials radiation properties, also need to be considered.

\section{A. Switching Devices}

Gas-filled thyratron devices have been used extensively in high-strength kicker designs. They have the advantage of a fast turn-on time, high peak and average power-handling capability, and radiation tolerance. However, since they are strictly a closing switch, their circuit and system functionality is limited.

With recent advancements in power-handling capability, devices such as IGBTs and MOSFETs are becoming more useful. These types of switching devices can act as both opening and closing switches, bringing new options into the high voltage modulator design. They have the advantages of functional flexibility, fast repetition rate, improved reliability, and improved future capability. Even with recent 
improvements in power-handling capability, however, large serial and parallel arrays are generally required for most kicker applications.

\section{B. Magnet Material}

The magnet material used in the fast kicker is usually high frequency ferrite. It can be used inside or outside of a vacuum chamber. Laminated magnetic alloys, such as METGLAS, can also be used as kicker magnet material outside of vacuum lines. However in high radiation areas, the insulation material used between laminations should be carefully selected for radiation tolerance.

\section{Multiple-Module VS. Single Module}

Kicker systems comprised of multiple modules benefit from reduced magnet-inductance requirements and lowered modulator voltage and current ratings. Additionally, the spare-parts inventory is reduced from a complete system to a single subsystem. Multiple modules can be used in many combinations to improve the overall system performance. For example, when each one can be triggered independently, or delayed differently from a central trigger, the resulting pulse will be a summation of all pulses, or a sparse pulse chain. In the event of a faulty module, the remaining modules can be tuned up accordingly to achieve the required total output thereby increasing system availability.

When multiple modules are used, the load magnet inductance per section is equivalent to the total inductance divided by the number of sections. However, the ratio of the stray inductance to magnet inductance will be increased, especially for the magnets residing inside the target chamber due to the feed-through inductance. This may lead to low power efficiency. Hence, in the design, the number of modules to be used and the overall efficiency should be balanced to obtain the maximum benefit. The use of solid-state power electronic devices and the desire to place magnets outside the vacuum chamber have made the modular approach more and more attractive.

\section{Grounding \& Shielding}

Since the kicker systems usually have the highest voltage in the accelerator and operate at very high frequencies, signal leakage can affect many other systems such as beam instrumentation, controls, lowlevel RF systems, and even its own control and auxiliary systems. Depending on the overall accelerator grounding design, the kicker vacuum chamber may or may not be isolated from rest of the ring. In either situation, a kicker system design with a low impedance signal return is beneficial. For multimodule systems, using a star-type grounding design can effectively reduce channel interference and eliminating internal ground loops. The proper shielding of modulators, including signal and output cables, will reduce the high frequency pulse radiation.
Fiber-optic isolation has also been used widely in kicker system designs.

\section{E. High-Intensity Beam Coupling}

A high-intensity beam can interact with a kicker magnet [3], and produce undesired effects such as beam losses and instabilities. It also poses a potential danger to the kicker system itself. With high-current beams of tens or even hundreds of amperes, this effect cannot be ignored.

One particular problem is that the beam passing through kicker magnet aperture can become an active, high-power current source. The power produced from this interaction must be dissipated in the power supply components and other magnets and can be high enough to cause component damage and trigger instabilities. To prevent these effects, the kicker system design should include a proper image current path, a low beam-impedance kicker structure, and increased component power ratings and magnet cooling.

\section{F. Radiation Concerns}

The injection and extraction regions of an accelerator tend to be heavy beam-loss areas. In highintensity proton accelerator and accumulator rings, the radiation level can be very high and prohibit access even after a long cooling time. This causes a very serious safety concern. To minimize the radiation exposure, new designs should have minimal components in the ring. This should make the system easier to maintain and reduce system down time. It will also allow a much wider selection of system components and materials such as solid-state power devices, oil-filled devices, and radiation sensitive materials.

\section{G. Cost Balance}

The construction cost of a high-strength kicker system is typically in the multi-million dollar range. However, current costs for operating large facilities including user costs, are getting higher due to complexity of the experiments. A balanced approach, with initial investments in R\&D and system design, will result in overall long-term cost savings.

\section{REFERENCES}

1. W. Zhang, et al., "A New Conceptual Design of the SNS Full Turn Fast Extraction Kicker Power Supply System", PAC 2001, June1822, 2001.

2. W. Zhao and M. Tigner, "Handbook of Accelerator Physics and Engineering”, World Scientific, 1998.

3. W. Zhang, et al., "Beam Coupling Phenomena in Fast kicker Systems", PAC 2001, June18-22, 2001. 